\title{
Evolución de las representaciones de lo femenino y de lo materno en psicoanálisis: dos paradigmas clásicos y uno emergente ${ }^{1}$
}

\author{
David Figueirôa ${ }^{2}$ \\ PsiRelacional, Lisboa, Portugal
}

\begin{abstract}
El autor pretende evidenciar los cambios en las representaciones de lo femenino y de lo materno en la teoría psicoanalítica. Identifica 3 diferentes paradigmas teóricos, formados en diferentes momentos de la evolución del psicoanálisis, usando como criterio diferenciador lo que designa como "estatuto ontológico del otro". Parte de dos paradigmas clásicos, el de la teoría freudiana ("MadreObjeto") y el de la teoría de las relaciones de objeto ("Madre-Función"), para llegar a un tercer paradigma, que emerge con psicoanálisis relacional, el de la teoría intersubjetiva, de Jessica Benjamin ("Madre-Sujeto"). También busca evidenciar la influencia cultural y social en la construcción de la teoría psicoanalítica pero, sobre todo, el sentido inverso de esa influencia, es decir, el potencial transformador que la teoría psicoanalítica ofrece, no sólo en la esfera individual, sino también en la esfera cultural y social. A este nivel destaca la teoría intersubjetiva de Benjamín como ejemplo de la articulación entre lo individual y lo social y su potencial transformador, en ambas esferas, a partir de la relación analítica
\end{abstract}

Palabras clave: Femenino, Materno, Otro, Reconocimiento mútuo.

The author intends to highlight the changes in the representations of the feminine and the maternal in psychoanalytic theory. He identifies 3 different theoretical paradigms, formed in different moments of the evolution of psychoanalysis, using as differentiating criterion what he denominates by "ontological status of the other". Two classic paradigms, the Freudian theory ("Mother-Object") and the school of object relations ("Mother-Function"), and a third paradigm that emerges with relational psychoanalysis, that of intersubjective theory by Jessica Benjamin ("Mother-Subject"). It also points to the cultural and social influence in the construction of psychoanalytic theory and the inverse direction of this influence, that is, the transformative potential that psychoanalytic theory offers not only in the individual sphere but also in the cultural and social sphere. At this level he emphasizes Benjamin's intersubjective theory as an example of the articulation between the individual and the social and its transforming potential, in both spheres, from the analytical relationship.

Key Words: Feminine, Maternal, Other, Mutual recognition.

English Title: Evolution of representations of the feminine and the maternal in psychoanalysis: two classic paradigms and one emerging.

\section{Cita bibliográfica / Reference citation:}

Figueirôa, D. (2019). Evolución de las representaciones de lo femenino y de lo materno en psicoanálisis: dos paradigmas clásicos y uno emergente. Clínica e Investigación Relacional, 13 (1): 97108. [ISSN 1988-2939] [Recuperado de www.ceir.info] DOI: 10.21110/19882939.2019.130107

\footnotetext{
${ }^{1}$ Trabajo presentado en las // Jornadas de Psicanálise Relacional. Fac. Psicologia, Lisboa, 17-19 de Mayo de 2018. Versión castellana preparada por el autor, con revisiones editoriales menores a cargo de la redacción de CelR. 2 Psicoterapeuta psicoanalítico, PsiRelacional - Associação de Psicanálise Relacional, Lisboa, Portugal; davidf@netcabo.pt
} 


\section{Freud: La madre-objeto de las pulsiones, una madre indiferenciada en sus atributos reales}

Uno de los legados de Freud fue haber contribuido a traer la sexualidad femenina al estudio científico. Sin embargo, su valor fue mitigado por la naturaleza falocéntrica de su teoría, que le proporcionó tantas - y justas - críticas.

La polémica cuestión de la "envidia del pene" concentra, simbólicamente, lo esencial de la disposición freudiana en cuanto a la posición relativa de lo femenino frente a lo masculino. Para Freud, el complejo central del desarrollo es el Edipo, donde, en su etapa inicial, la percepción del pene (o de su falta) es el criterio en torno al cual giran los principales anhelos y angustias en el niño y en la niña. Si la angustia masculina por excelencia es la de la castración (el miedo a perder el pene), el complejo femenino determinante es el de la envidia del pene (con la fantasía de haber quedado sin pene), véase, por ejemplo, Freud, 1908.

Esta centralidad del pene y de lo masculino en Freud se expresa también en la centralidad del Padre como agente de desarrollo. Esta centralidad viene desde el padre primitivo de "Totem y Tabú" (1913), que después de asesinado y comido por los hijos, es recuperado mediante el Totem, las acciones que constituyen, en este mito psicoanalítico, las bases de la organización social, de la moral y de la religión. También está representado por el nacimiento del padre edípico, lo odiado se transforma en un agente de "prohibición" y "ley", con su papel decisivo en la represión de la fuerza instintiva básica en lo humano y, por lo tanto, salvaguardando la civilización.

La centralidad fálica en Freud se expresa, por ejemplo, en las controvertidas correspondencias psicológicas entre lo masculino y lo "activo" y lo femenino y lo "pasivo" (Freud, 1918, citado por Quinodoz, 2004, p.200).

Las líneas de fuerza del pensamiento freudiano inscriben a la mujer como objeto del deseo (y al hombre como sujeto del deseo). La mujer representa la tentación o la cercanía con lo pulsional, en función de la urgencia sexual que mueve al hombre - y el niño (que es "padre del hombre"), en su complejo edípico. Si la madre es el objeto del deseo, el padre se constituye como el prohibidor, el castrador $y$, de esa forma, el libertador que se opone al deseo pulsional infantil $y$, implícitamente, a su propio deseo pulsional. No es difícil de reconocer cómo Freud, con su genio psicoanalítico revolucionario, no dejó de estar condicionado por la cultura patriarcal vigente en la época y con raíces profundas en la 
historia cultural europea (como todos sabemos, la mujer nace del hombre, "Eva, de la costilla de Adán").

En Freud, la presencia "real" de la madre con funciones cuidadoras es poco diferenciada: la relación madre / bebé gira en torno a la cuestión de la gratificación / frustración pulsional y en la cuestión derivada de la proximidad madre-bebé, esfera de la vida pulsional, y su destino sano: la separación madre-bebé, con la posibilidad de renuncia y sublimación pulsional. El agente de la separación, el agente del desarrollo psíquico es el padre.

En este sentido, hay una coherencia significativa entre la predominancia masculina y paterna en la religión (Jesús es hijo de Dios, Jesús es Dios, Jesús es hombre, Dios es evidentemente masculino, como los sacerdotes son hombres, etc.), en las estructuras patriarcales en la sociedad (el padre de familia, el señor de la tierra, la sucesión por vía masculina, etc., etc.) y la concepción psicoanalítica inicial.

Selecciono un pasaje de Freud que ilustra la intersección de los paradigmas cultural y psicoanalítico sobre lo femenino y lo materno, de "Malestar en la Civilización" (1930), por lo tanto ya en la fase consolidada de su obra:

"Las mujeres representan los intereses de la familia y de la vida sexual [en oposición a los intereses más latos de la civilización]. El trabajo de civilización se ha convertido cada vez más en un asunto masculino, enfrentando a los hombres con tareas cada vez más difíciles y obligándolos a realizar sublimaciones instintivas de las que las mujeres son poco capaces. Ya que el hombre no dispone de cantidades ilimitadas de energía psíquica, tiene que realizar sus tareas realizando una distribución apropiada de su libido. Lo que emplea para fines culturales, en gran parte lo extrae de las mujeres y de la vida sexual. Su constante asociación con otros hombres y la dependencia de sus relaciones con ellos lo alienan incluso de sus deberes de marido y de padre. De esa manera, la mujer se descubre relegada a segundo plano por las exigencias de la civilización y adopta una actitud hostil hacia ella" (p.109).

En este caso, el hombre activo, agente de la civilización, capaz de sublimar las pulsiones, y la mujer, vista por Freud, y por tanto, contemplada por el hombre, como representante de la familia y de la vida sexual, rehén y guardián de una pulsionalidad endógena, que se opera haciéndose objeto materno y objeto sexual.

Tenemos, por lo tanto, un paradigma psicoanalítico inicial sobre lo femenino y sobre lo materno caracterizado por la atribución a lo femenino de la proximidad con lo pulsional, en el carácter de objeto, y de esa forma constituyéndose como una oposición a la civilización y al desarrollo psíquico decisivo. En Freud, la gran tarea del desarrollo psíquico es, por lo 
tanto, la capacidad de renuncia en relación a lo pulsional, la capacidad de sublimación pulsional, y, consecuentemente, la tarea relacional primordial es la separación con la madre.

Coherentemente, en Freud, la madre real surge indiferenciada, no se le conocen atributos específicos. Ella es, para su bebé, nada más que el modelo de todo el amor futuro (1930, p.89). Este enlace pulsional con el objeto, cuando de él se consigue separar (cuando no se establece la vía psicótica), será la reminiscencia infantil de que el amor adulto se alimenta. La indiferenciación materna está aquí de la mano con una idealización reductora del materno real.

La gran cuestión del desarrollo es posterior y la figura en torno a la cual se procesará, será el padre. El psicoanálisis paterno, edípico, civilizador, de Freud dejó a la mujer y a la madre no lejos del papel que les competía en la cultura más amplia. Pero abrió un camino decisivo para avances posteriores de enorme impacto, llegando rápidamente a constituirse un nuevo paradigma duradero sobre lo femenino y, sobre todo, sobre lo materno.

\section{Relaciones de Objeto - La madre-función "suficientemente buena"}

Inspirada en Sàndor Ferenczi y en la importancia de la madre real, en el descubrimiento del psicoanálisis infantil, por Anna Freud y Melanie Klein, y en los hallazgos de la psicología del desarrollo, la escuela británica de las relaciones de objeto trajo este nuevo paradigma. Desde luego, autores tan prominentes como Melanie Klein y Donald Winnicott reivindicaron el lugar autónomo de la sexualidad femenina, con críticas manifiestas a la teoría freudiana que la hacía dependiente de la sexualidad masculina. "La envidia del pene como fuente de motivaciones poderosas en la niña y en la mujer no puede ser ignorada [concediendo aquí a Freud, pero añadiendo], pero a pesar de ello existe sin duda una fantasía y una sexualidad femenina básica, que tienen su origen en la más remota infancia" (1988, p.63-64), y explícita como el carácter activo de la sexualidad femenina, el carácter activo de la "vagina" es camuflado por fantasías "del orden del recogimiento, del guardar en secreto, del esconder", en coherencia con la propia disposición anatómica. Y añadimos, en coherencia también con la disposición cultural, que la familia y la educación reproducen.

Es la escuela de las relaciones de objeto la que promueve en definitiva el paso del foco del Edipo tardío hacia la relación primitiva con la madre. Klein, Fairbairn, Winnicott, Balint, Bowlby, Bion, etc. van a ampliar la mirada psicoanalítica sobre la relación primaria y van a hacer emerger en el pensamiento psicoanalítico la importancia de la relación madre-bebé. 
La mirada sobre el bebé y sobre la relación madre-bebé encuentra tal vez en Winnicott su máxima expresión cuando afirma que "no existe tal cosa llamada bebé" (1957, p.99), refiriéndose a la absoluta dependencia de éste en relación a la madre. Lo que existe es la "unidad madre-bebé". Pero aquí ya no estamos en el marco de la fuerza y del control pulsional como criterio fundamental para el desarrollo psíquico, sino en el marco de la emergencia del self individual, frente a la dependencia absoluta inicial con la madre.

Es, por lo tanto, en torno a la figura materna y de la relación primaria, que se desarrolla la teoría psicoanalítica, relegando al padre freudiano hacia un momento posterior, un momento ya de menor expresión e impacto.

La madre se vuelve progresivamente más diferenciada. A partir de las "buenas experiencias" que la madre real, evocada por Melanie Klein, debe proporcionar a su bebé, el psicoanálisis fue desvelando y caracterizando un conjunto de atributos y de funciones maternas cada vez más apuradas - desde entonces el psicoanálisis y la psicología del desarrollo han sido prolíficas en su identificación, como la función continente de Bion, o la función vinculante de Bowlby o la de "mentalización" de Fonagy, por ejemplo. EI psicoanalista de segunda generación que dió mayor amplitud a las funciones maternas fue Donald Winnicott, a partir de su inmensa experiencia como pediatra en la observación de los bebés con sus madres. Winnicott describió como función materna esencial la de la continuidad relacional en el tiempo, y delineó, dentro de esa continuidad cuidadora, las funciones de holding (sostener al bebé) y handling (el manejo físico e psiquico), la función de dejarse descubrir y crear por el bebé, las funciones de no indiferencia y de no intrusividad, las funciones de presentación del mundo al bebé, de la sustentación de las paradojas inherentes al objeto y al espacio transicional, del juego o aún las funciones de no retaliación, de no retirada y de supervivencia a la pulsionalidad del niño, entre otras (véase, por ejemplo, 1984, 1987).

Winnicott descubrió definitivamente la madre real, que se vino a revelar una super-madre (su descubrimiento representa también un reconocimiento de la super-tarea que las madres desarrollaban sin el conocimiento consciente de los hombres y del propio psicoanálisis hasta entonces). Pero esta super-madre es una madre sobrecargada con una responsabilidad extraordinariamente exigente (e inevitablemente culpabilizada por las fallas), lo que es algo que el propio Winnicott busca aliviar con su realista y equilibrada síntesis de las funciones maternas: la madre está condenada a "fallar, a fallar, y a fallar", sin que ello comprometa el desarrollo, si es "suficientemente buena". Pero, antes de eso, en las primeras semanas de vida del bebé, como lo afirma, es deseable que la madre ofrezca una adaptación perfecta a las necesidades del bebé. 
La "preocupación maternal primaria" ayudará a la madre, pero la función cuidadora no podrá ser soportada sólo por ella, e implicará el padre y, progresivamente, todos los elementos significativos de la familia, de la escuela y de la comunidad, incluyendo, cuando se les llama, los psicoterapeutas. Todos los elementos significativos del medio formarán parte de la "madre suficientemente buena" que representa a todos $(1957,1984)$. Winnicott, de cierta forma, preconiza una sociedad en la que todos tenemos responsabilidades sobre todos los niños (Figueirôa, 2014).

Esta valoración y diferenciación de la madre real es acompañada por el desplazamiento del foco psicoanalítico de lo paterno a lo materno, del masculino represor / liberador a el femenino cuidador. De la función de la autoridad a la función del cuidar. De la educación al desarrollo emocional. La madre "suficientemente buena" es una madre real, ampliamente diferenciada al nivel funcional, y ahí se incluye la "relacionalidad" (Greenberg y Mitchell, 1983, p.227-250). El desarrollo libidinal y pulsional se inscribe desde entonces en el más amplio desarrollo emocional del niño.

Este nuevo paradigma materno, que, en la senda de Winnicott, podemos designar también de "madre suficientemente buena", incluyendo todas las funciones psicoanalíticamente discriminadas, pone énfasis en la madre real y en las funciones maternas y permanece como el más representativo en la actualidad. Esta "madre suficientemente buena" es desde entonces la nueva matriz teórica del desarrollo psíquico, que abre camino al padre y a las demás relaciones. El niño debe encontrar una "madre suficientemente buena" para poder constituirse a sí misma como unidad autónoma, con su self único, diferenciado y creativo.

La "madre objeto" freudiana pasa a ser una madre-sujeto de funciones, pero que se mantiene, en la perspectiva del niño, como un objeto proveedor a sus necesidades.

En la transición de los paradigmas, pasamos igualmente, del modelo de la fijación libidinal para modelo de la falla del desarrollo, poniendo a la relación analítica la tarea de suplir lo que quedó en falta (Greenberg y Mitchell, 1983, p.451-462). El psicoanálisis freudiano, neutral, interpretativo y orientado al conocimiento (hacer el inconsciente consciente) evolucionó así en el sentido de la inclusión de la importancia de la relación transformadora, proveedora de los elementos relacionales que faltan. El analista freudiano desvelador del inconsciente fue complementado por el analista como figura significativa en la "regresión a la dependencia" en autores posteriores (Ferenczi o Winnicott, por ejemplo, 1954). El analista neutral fue llamado a la relación. El analista freudiano objeto de la transferencia (en su dimensión esencialmente "femenina") y sujeto del saber y de la interpretación (en su dimensión esencialmente "masculina") evolucionó, por lo tanto, en el sentido de la inclusión de funciones relacionales diversificadas. La articulación en el interior del analista 
y en la relación clínica de estas valencias interpretativa y relacional constituye desde entonces el mayor desafío teórico-clínico a la actitud psicoterapéutica (por ejemplo, Winnicott 1955-56), un desafío que se ha extendido hasta la actualidad.

\section{Teoría intersubjetiva de Benjamin: la madre-sujeto, centro de subjetividad}

En los años 8o los movimientos relacionales (Greenberg y Mitchell, 1983, y compañeros, vd. Mitchell y Aron, 1999) y intersubjetivistas (por ejemplo, Orange, Atwood y Stolorow, 1993) revolucionaron el psicoanálisis con el llamado "giro relacional". Estos autores profundizaron la autonomía de la mirada sobre lo femenino, relanzaron las cuestiones de género en el debate psicoanalítico y ampliaron el paradigma de la "madre suficientemente buena", valorando la importancia de los contextos intrapsíquicos e interpersonales, familiares, sociales y culturales.

Jessica Benjamin, en el corazón del movimiento relacional norteamericano, es una autora feminista que formuló una teoría original de la intersubjetividad como una adquisición del desarrollo (1990, 2018).

Ella se asienta en la percepción del otro no sólo como entero y separado de mí, sino como sujeto de una subjetividad y de una intencionalidad propia e independiente de mí. En la relación con la madre -o con el cuidador- esta capacidad se desarrolla a partir de la propia capacidad de la madre de reconocer a su bebé mientras un ser diferenciado (lo que el propio bebé suscita con sus aptitudes expresivas y relacionales), su capacidad para sentirse y asumir como un ser diferenciado en relación a su bebé, proporcionando al bebé la posibilidad de constituir una relación con una madre que es un sujeto y un centro de experiencia y subjetividad propias.

Cuando las cosas marchan bien, madre y bebé articulan progresivamente una relación de reconocimiento mutuo, en la que la acción del otro no es meramente referenciada a las necesidades de relación de objeto internas del primero.

Somos, por lo tanto, en un patrón relacional evolutivo, en un grado máximo de relacionalidad (Mitchell, 2000), que da cuerpo al paso de una relación sujeto-objeto hacia una relación sujeto-sujeto, adquirida en el desarrollo, teniendo como momento crítico el correspondiente a la "fase de reaproximación" de Margaret Mahler, por los 2 años de edad, que Benjamin reinterpreta como una "crisis de crecimiento" de la intersubjectividad (1990, p.190).

Una de las bases del pensamiento de Benjamin puede ser encontrada, como ella misma asume, en la intersubjectividad winnicotianna, expresada en el texto "El uso de un objeto y 
la relación a través de identificaciones" (1971) en el que Winnicott establece la diferencia entre la capacidad de relación de objeto - esta siendo subjetivada y auto-referenciada al mundo interno, es decir, a la implicación del otro real en el escenario interno preexistente - y la capacidad de usar el objeto, de usar el otro real, lo que presupone, para Winnicott, el reconocimiento del otro real como independiente del "otro" que hay en mí. Para Winnicott esta adquisición depende de la capacidad de supervivencia del otro real al movimiento destructivo del sujeto en relación a él. Cuando el otro sobrevive a la destrucción fantasiosa, inherente a la vida psíquica, establece entonces las condiciones para ser descubierto como objeto real -o, como diríamos hoy, como um sujeto otro.

Para Benjamin, la capacidad de reconocimiento mutuo no es una adquisición plena, porque implica una permanente tensión entre la subjetivación omnipotente, con la negación y la destrucción del otro, y el reconocimiento de la realidad del otro, con su supervivencia. En nuestra mente estamos, de forma sistemática y dinámica, perdiendo y recuperando esa capacidad, en una tensión permanente entre destrucción y supervivencia, subjetivación y realidad, relación de objeto interna y usufructo del otro real, entre la auto-referencia intrapsíquica y el reconocimiento del otro.

Conocemos esta dinámica en nuestras relaciones cotidianas e íntimas, con nuestros padres, hijos, jefes, amigos o compañeros amorosos. Cuando la cosa aprieta, cuando el conflicto hace peligrar la seguridad de nuestro self, la tendencia psíquica, defensiva, es leer la realidad del otro en función de mi propia subjetividad. A veces, llevamos tiempo hasta la restauración del reconocimiento del otro.

El psiquismo, para Benjamín, no es armonioso. Estamos siempre entre la ruptura de la intersubjetividad y la reparación y restauración de la misma. La capacidad intersubjetiva se traduce, por lo tanto, en la capacidad de sostener esta tensión y de repararla cuando entra en ruptura.

Ahora bien, como dijimos atrás, es en la relación madre-bebé, o cuidador-bebé, que se desarrolla esta capacidad. Lo que implica la capacidad de aceptación, por la madre y por el bebé, que la madre-función coexiste con la madre-sujeto, con la mujer que existe por sí misma y que tiene vida más allá del hijo.

Esto implica que la teoría de las relaciones de objeto, fundada en lo intrapsíquico, se mantiene válida, dado el fondo de relaciones de objeto interno que se mantiene activo hasta en el psiquismo más evolucionado, pero debe añadirse a una teoría intersubjetiva que representa la posibilidad de liberación de las relaciones de objeto internas y de la posibilidad de reconocimiento mutuo. 
Cuando predominan las relaciones de objeto internas, el otro es la cara complementaria de lo que me angustia, es decir, es el otro que me hace sentir lo que siento, por eso, lo ataco, disminuyéndolo y destruyéndolo como equivalente a mi. El restablecimiento de la capacidad de reconocimiento mutuo es lo que permitirá la transición de la lucha de poder al reparto del poder, de la desigualdad a la igualdad, del amor narcisista al amor maduro. El reconocimiento mutuo instituye la posibilidad de descubrir el otro real - $y$, alli, también la posibilidad de ser descubierto como si.

La teoría de la intersubjetividad de Benjamin fue también un paso adelante en el psicoanálisis feminista, al evidenciar las bases psíquicas de la estructura de dominación masculina en la sociedad. La madre (interna) omnipotente, que todo puede ofrecer o negar al niño es la figura central de la teoría de las relaciones de objeto. La madre todopoderosa de la dependencia infantil se mantiene siempre en las bases del psiquismo adulto. La dominación social masculina, las estructuras patriarcales y machistas, se desarrollan como contrapoder a esta madre omnipotente y poderosa, de la que el niño interno depende y de la que necesita liberarse para poder existir. Cuando no se sustenta la capacidad intersubjetiva, que es esencial para el reconocimiento de la mujer en la madre y la constitución de la relación con lo femenino autónomo, se mantiene el sujeto prisionero, en la relación con lo femenino, de esta base de relación de objeto, y precisa de organizar el poder de someterla. Va de la sensación de dependencia a la sujeción del otro. Esta será, además, la base de todas las relaciones de poder y sumisión, desde el punto de vista de la intersubjetividad de Benjamín. Una visión alternativa, o complementaria, a la de Donald Meltzer y sus trabajos sobre tiranía y sumisión (1968).

En este sentido, la teoría de Benjamin no sólo incorpora la evolución social de emancipación de la mujer a lo largo del siglo XX y hoy, sino que proporciona una comprensión de las bases intrapsíquicas de la dominación masculina e identifica una adquisición psicológica y relacional fundamental para que el reconocimiento mutuo entre mujeres y hombres pueda evolucionar dinámicamente de una lucha de poder y sumisión para un reparto del poder - en la familia y en la sociedad.

Ahora bien, lo que pretendo mostrar aquí es que esta teoría trae nuevos elementos para un nuevo paradigma psicoanalítico contemporáneo sobre lo femenino y lo materno en el contexto del psicoanálisis relacional. Un paradigma basado en una evolución del estatuto ontológico del Otro. En particular, la mujer encuentra en la teoría intersubjetiva su plena emancipación como sujeto, siendo que el mayor alcance de esa conquista se hace dentro del "lugar sagrado" de la madre. Si la madre nació (y es necesario que nazca -como nos recordó Matos, 2019) y se constituyó sobre lo femenino, éste puede mantenerse vivo más 
allá de lo materno. Por lo demás, si esto no sucediera desde luego, si la simbiosis o la fusión o la identificación con el bebé fuera perfecta, la madre haría como el bebé y comería al bebé, como señala Lacan, en su crítica a la teoría de las relaciones de objeto y al amor primario (1975, citado por Benjamin, 2018, p.27).

Cuando falla la capacidad intersubjetiva en la madre, la madre constituye el bebé como una prolongación narcisista y se vacía de su subjetividad a través de la idealización del bebé, cristalizando el nacimiento de ambos como si de uno solo se tratara. En la clínica encontramos varias expresiones de la falta de reconocimiento, desde las madres con perturbaciones narcisistas severas a las madres con funcionamiento neurótico, predominantemente preocupadas y controladoras y/o evitantes de la intimidad emocional con el niño real.

Es, por lo tanto, en el interior de la propia madre que la teoría reconoce a la mujer, siendo además esa afirmación de lo "femenino que está más allá de lo materno" un componente constituyente de lo propio materno. La madre-objeto de Freud y la madre-función de la teoría de las relaciones de objeto son complementadas - pero no sustituidas - por la madresujeto de la teoría intersubjetiva, constituyendo ésta una nueva dimensión de la "imago" materna en construcción en la teoría psicoanalítica. En el caso de la madre, la madre-sujeto representa así una conquista psíquica (en el desarrollo), una conquista teórica (en la teoría psicoanalítica), una conquista clínica (como la inclusión de la subjetividad del terapeuta, la sustentación de la tensión intersubjetiva y del "tercero" y de la capacidad de reparar la destrucción) y una conquista social (en el reconocimiento de lo femenino).

En otro trabajo (Figueirôa, 2016) mencioné la evolución del papel del analista en términos semejantes a la evolución de los paradigmas sobre la madre: del analista-objeto al analistafunción al analista-sujeto, defendiendo que cada una de las adquisiciones no sustituye a la anterior, antes viene a añadir y complementar la anterior. Lo mismo ocurre con la madre:

Las responsabilidades maternas - o de los cuidadores primarios - incluyen 1) la capacidad de ofrecerse, como objeto, para las necesidades pulsionales y emocionales del bebé, 2) la capacidad de asegurar las diferentes e importantes funciones que corresponden a las necesidades evolutivas del niño en sus diferentes etapas de crecimiento y 3 ) la capacidad de ser quienes son y reconocer a su hijo como es y puede llegar a ser, con la articulación progresiva del reconocimiento mutuo y de la capacidad intersubjetiva.

Concluyo, diciendo que la exigencia sobre la madre y sobre el padre y sobre todos los cuidadores significativos, en la familia, en la escuela y en la comunidad, implica, más que un saber técnico y objetivo, una afirmación firme de la subjetividad responsable de cada 
interviniente, y la valorización de la subjetividad y de la responsabilidad progresiva del niño -que un día sera adulto, ciudadano y cuidador de la futura generación.

\section{REFERENCIAS}

Benjamin, J. (1990). Recognition and Destruction: An Outline of Intersubjectivity. Relational Psychoanalysis: the Emergence of a Tradition (ed. Mitchell, S. e Aron, L.). NY: Routledge, 1999.

Benjamin, J. (2018). Beyond Doer and Done To: Recognition Theory, Intersubjectivity and the Third. NY: Routledge.

Figueirôa, D. (2014). A Criança e o Mundo Exterior: Onde Está o Adulto? Clínica e Investigación Relacional, 8 (2): 481-488. Madrid: Ágora Relacional.

Figueirôa (2016). Relação, Subjectividade, Contexto: Organizadores da Teoria e da Clínica Relacionais (comunicação pessoal, I Jornadas da PsiRelacional).

Freud, S. (1908). Sobre as Teorias Sexuais Infantis. Edição Standard Brasileira das Obras Psicológicas Completas de Sigmund Freud, Vol. IX (pp.189-204). Rio de Janeiro: Imago, 1996.

Freud, S. (1913). Totem e Tabu. Edição Standard Brasileira das Obras Psicológicas Completas de Sigmund Freud, Vol. XIII (pp. 13-162). Rio de Janeiro: Imago, 1996.

Freud, S. (1918). História de Uma Neurose Infantil. Edição Standard Brasileira das Obras Psicológicas Completas de Sigmund Freud, Vol. XVII (pp.15-127). Rio de Janeiro: Imago, 1996.

Freud, S. (1930). O Mal-Estar na Civilização. Edição Standard Brasileira das Obras Psicológicas Completas de Sigmund Freud, Vol. XXI (pp.67-148). Rio de Janeiro: Imago, 1996.

Greenberg, J. e Mitchell, S (1983). Relações de Objecto na Teoria Psicanalítica. Lisboa: Climepsi, 2003.

Matos, M (2019). O Nascimento da Mãe no contexto da Perinatalidade. Clínica e Investigación Relacional, 13 (1). Madrid: Ágora Relacional.

Meltzer, D. (1968). La Tyrannie. Psychanalyse du Lyen Tyrannique (Ciccone et al). Paris: Dunod, 2003.

Mitchell, S. e Aron, L. (1999). Relational Psychoanalysis: the Emergence of a Tradition. NY: Routledge.

Mitchell, S. (2000). Relationality: From Attachment to Intersubjectivity. Psychology Press, 2004.

Quinodoz, J-M (2004). Ler Freud. Porto Alegre: Artmed, 2007.

Winnicott, D. (1954; 1958). Aspectos Clínicos e Metapsicológicos da Regressão no Contexto Psicanalítico. Da Pediatria à Psicanálise. Rio de Janeiro: Imago, 2000.

Winnicott, D. (1955-56; 1958). Formas Clínicas da Transferência. Da Pediatria à Psicanálise. Rio de Janeiro: Imago, 2000. 
Winnicott, D. (1957). A Criança e o Seu Mundo. Rio de Janeiro: Zahar, 1971.

Winnicott, D. (1971). O Uso de Um Objecto e o Relacionamento através de Identificações. $O$ Brincar \& a Realidade. Rio de Janeiro: Imago, 1975.

Winnicott, D. (1984). Privação e Delinquência. São Paulo: Martins Fontes, 2002.

Winnicott, D. (1987). Os Bebés e Suas Mães. São Paulo: Martins Fontes, 2002.

Winnicott, D. (1988). Natureza Humana. Rio de Janeiro: Imago, 1990.

Original recibido con fecha: 26/9/2018 Revisado: 15/03/2019 Aceptado: 30/03/2019

En este mismo número de CeIR se publica online la versión original portuguesa de este trabajo. 